\title{
Examination of the Shape and Structure of (111)-oriented GaAs Tensile-Strained Quantum Dots using Transmission Electron Microscopy, Electron Energy Loss Spectroscopy, and Atom Probe Tomography
}

Kevin A. Grossklaus ${ }^{1 *}$, Christopher F. Schuck ${ }^{2}$, Paul J. Simmonds ${ }^{2,3}$, and Thomas E. Vandervelde ${ }^{1}$

${ }^{1 .}$ Department of Electrical and Computer Engineering, Tufts University, Medford, MA, USA.

2. Micron School of Materials Science \& Engineering, Boise State University, Boise, ID, USA.

3. Department of Physics, Boise State University, Boise, ID, USA.

* Corresponding author: kevin.grossklaus@tufts.edu

Researchers have extensively studied and used semiconductor quantum dots (QDs) as dislocation free and optically tunable nanostructures in a variety of optoelectronic applications. In particular, compressively-strained InAs QD self-assembly via the Stranski-Krastanov (SK) growth mode on (001)oriented GaAs substrates has been thoroughly studied [1], and has enabled the creation of high efficiency quantum dot lasers. However, SK self-assembly of QDs on (001)-surfaces has been predominantly limited to compressively-strained systems, thus limiting the number of QD/matrix material combinations available. The growth of tensile-strained GaAs quantum dots (TSQDs) on InAlAs(111)A surfaces has recently been demonstrated [2][3]. The use of tensile strain to drive the selfassembly process allows us to invert the QD/matrix lattice constant requirement. Early studies of this new TSQD system show interesting structural and optical properties from both the QDs and the wetting layer (WL) from which they form. However, the exact formation mechanism and structural properties of the TSQDs have not been extensively studied. To better understand and utilize GaAs TSQDs for optoelectronic applications, additional structural characterization is needed.

This study describes the transmission electron microscopy (TEM) and atom probe tomography (APT) characterization of epitaxial (111)-oriented GaAs WL and TSQDs grown on and capped by InAlAs. The materials studied were grown by solid source molecular beam epitaxy (MBE) on InP(111)A substrates, above which is grown an $\operatorname{In}_{0.53} \mathrm{Ga}_{0.47} \mathrm{As}$ smoothing buffer, followed by an $\operatorname{In}_{0.52} \mathrm{Al}_{0.48} \mathrm{As}$ bottom barrier. Consistent with the SK growth, GaAs deposition on this InAlAs initially forms a 2D WL, then at a critical thickness (2.5 ML for GaAs TSQDs) QDs form via strain-driven self-assembly. The sample set examined consists of TSQDs created by 2.5-4.5 monolayers (ML) of GaAs deposition. The TSQDs are then capped with an additional InAlAs top barrier. Finally, another 2.5-4.5 ML of GaAs is deposited to form TSQDs for surface analysis by atomic force microscopy. Cross-sectional GaAs TSQD samples were prepared by FIB lift-out and examined by TEM and scanning transmission electron microscopy (STEM) in a JEOL ARM 200F aberration corrected microscope operated at 200kV. Figure 1 shows high resolution TEM images of the QD layer location in two samples with different amounts of GaAs deposited. The TSQDs are difficult to image directly in both TEM and STEM imaging modes because of their very low z-contrast difference relative to the surrounding InAlAs matrix and low straincontrast from the WL. STEM electron energy loss spectroscopy (EELS) mapping was used to more clearly examine the TSQD size and the WL thickness. Figure 2 shows a high resolution STEM image and EELS elemental maps showing the location of a $2.5 \mathrm{ML}$ GaAs sample WL. WL thickness was found to increase with the amount of GaAs deposited for TSQDs creation. The exact shape and size of the QDs and the degree to which the GaAs intermixes with the surrounding matrix is also important for understanding the TSQDs' optical properties. The shape of the GaAs TSQDs and thickness of the WL 
around the QDs and between them was examined by APT using a Cameca LEAP 4000X HR. [4]

References:

[1] A.D. Yoffe, Adv. Phys. 50 (2001), 1-208.

[2] C.D. Yerino et al., Appl. Phys. Lett. 105 (2014), 251901.

[3] C.F. Schuck et al., J. Vac. Sci. Technol. B 36 (2018), 031803.

[4] The authors gratefully acknowledge support for this study from the National Science Foundation under NSF ECCS Grant No. 1806311 and NSF CAREER Grant No. 1555270. Microscopy was performed at the Center for Nanoscale Systems (CNS) at Harvard University, a member of the National Nanotechnology Coordinated Infrastructure Network (NNCI), which is supported by the National Science Foundation under NSF award No. 1541959.

a) 2.5ML GaAs

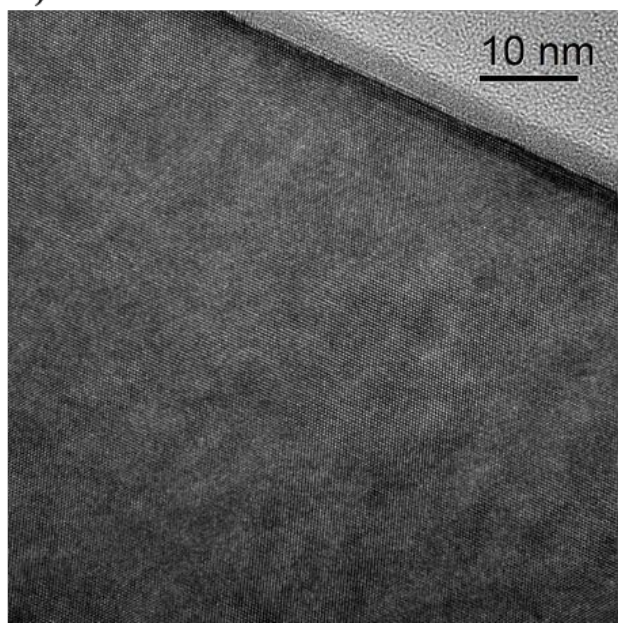

\section{b) 4.5ML GaAs}

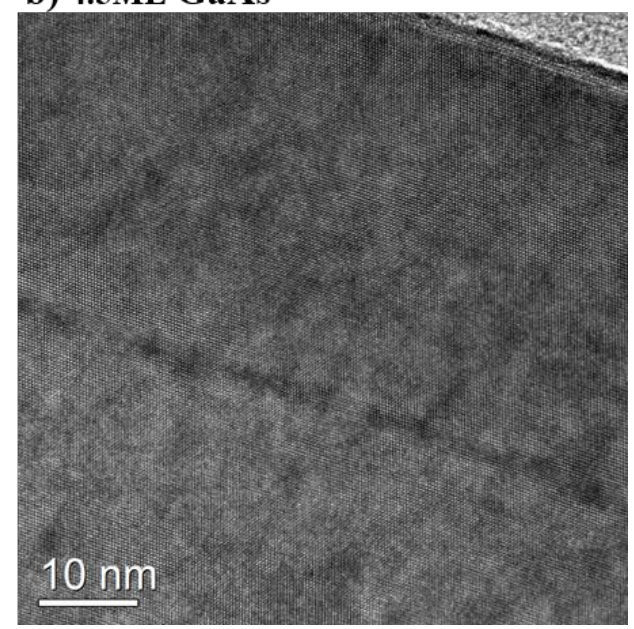

Figure 1. TEM images of a) 2.5 ML and b) 4.5 ML GaAs TSQD samples showing the QD layers at a depth of $\sim 40 \mathrm{~nm}$ below the material surface. In a) the contrast from the QDs and WL is extremely low.

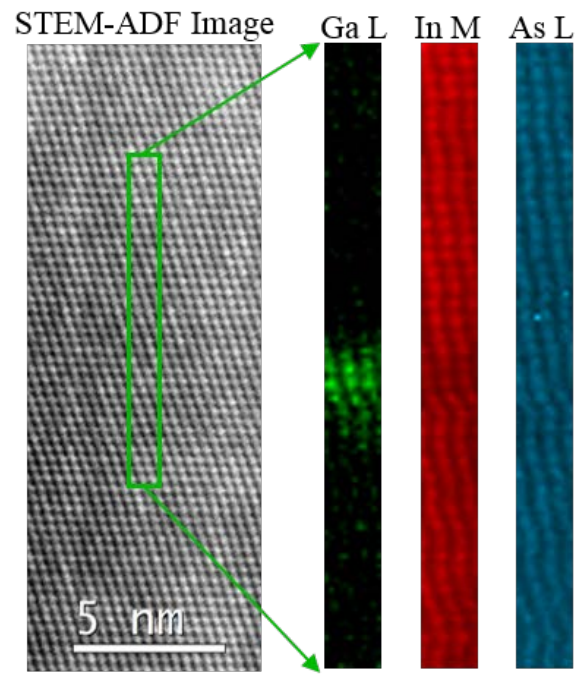

Figure 2. The left panel shows an ADF STEM image of a 2.5ML GaAs TSQD sample, with a green box indicating the area used for EELS mapping. To the right are Ga, In, and As EELS edge signals collected from mapping. Note that the WL not visible in Figure 1 a) can be seen in the Ga map. 